\title{
Fine-Tuning Pyridinic Nitrogen in Nitrogen-Doped Porous Carbon Nanostructures for Boosted Peroxidase-Like Activity and Sensitive Biosensing
}

\author{
Hongye Yan, ${ }^{1}$ Linzhe Wang, ${ }^{2}$ Yifeng Chen, ${ }^{1}$ Lei Jiao, ${ }^{1}$ Yu Wu, ${ }^{1}$ Weiqing Xu, ${ }^{1}$ Wenling Gu, \\ Weiyu Song $\oplus^{2}$, Dan Du $\oplus^{3},{ }^{3}$ and Chengzhou Zhu $\oplus^{1}$ \\ ${ }^{1}$ Key Laboratory of Pesticide and Chemical Biology of Ministry of Education, International Joint Research Center for Intelligent \\ Biosensing Technology and Health, College of Chemistry, Central China Normal University, Wuhan 430079, China \\ ${ }^{2}$ State Key Laboratory of Heavy Oil Processing, China University of Petroleum, Beijing 102249, China \\ ${ }^{3}$ School of Mechanical and Materials Engineering, Washington State University, Pullman, Washington 99164, USA
}

Correspondence should be addressed to Weiyu Song; songwy@cup.edu.cn and Chengzhou Zhu; czzhu@mail.ccnu.edu.cn

Received 13 July 2020; Accepted 14 September 2020; Published 6 November 2020

Copyright (c) 2020 Hongye Yan et al. Exclusive Licensee Science and Technology Review Publishing House. Distributed under a Creative Commons Attribution License (CC BY 4.0).

Carbon materials have been widely used as nanozymes in bioapplications, attributing to their intrinsic enzyme-like activities. Nitrogen $(\mathrm{N})$-doping has been explored as a promising way to improve the activity of carbon material-based nanozymes (CMNs). However, hindered by the intricate $\mathrm{N}$ dopants, the real active site of $\mathrm{N}$-doped CMNs (N-CMNs) has been rarely investigated, which subsequently retards the further progress of high-performance N-CMNs. Here, a series of porous $\mathrm{N}$-CMNs with well-controlled $\mathrm{N}$ dopants were synthesized, of which the intrinsic peroxidase (POD)like activity has a positive correlation with the pyridinic $\mathrm{N}$ content. Density functional theory calculations also reveal that pyridinic $\mathrm{N}$ boosts the intrinsic POD-like activity of N-CMNs. Pyridinic-N dopant can effectively promote the first $\mathrm{H}_{2} \mathrm{O}$ desorption process in comparison with the graphitic and pyrrolic $\mathrm{N}$, which is the key endothermic reaction during the catalytic process. Then, utilizing the optimized nanozymes with high pyridinic $\mathrm{N}$ content $\left(\mathrm{N}_{\mathrm{P}}-\mathrm{CMNs}\right.$ ) and superior POD-like activity, a facile total antioxidant capacity (TAC) assay was developed, holding great promise in the quality assessment of medicine tablets and antioxidant food for healthcare and healthy diet.

\section{Introduction}

Nanomaterial-based enzyme mimics, defined as nanozymes, have emerged and attracted considerable attention recently. As emerging substitutes of natural enzymes, nanozymes possess exceptional features including high stability, durability under harsh conditions, and simple preparation with low costs [1-9]. Carbon material-based nanozymes (CMNs), as a star family in nanozymes, have been widely explored in sensing, therapy, and catalysis, since they have been discovered to possess high operational stability against stringent conditions and could be prepared facilely with low costs [10-16]. However, as metal-free catalysts, pure CMNs have been endowed with moderate catalytic activities, which are still far from meeting the requirements of high catalytic activity. To address this issue, heteroatom $(\mathrm{N}, \mathrm{B}, \mathrm{P}, \mathrm{S}$, or Se) dop- ing is an efficient method to improve the catalytic activities of carbon materials [17-23]. Among them, N-doping has been extensively studied due to the fact that a similar atomic radius of nitrogen as carbon makes it easier to incorporate into the graphitic lattice [10, 24].

To date, $\mathrm{N}$-doped CMNs (N-CMNs) have shown great promise in biosensors, clinical diagnosis, and therapy, attributing to their intrinsic peroxidase (POD)-like activities [10-13]. Although great progress has been made in this field, further verification of active sites and in-depth understanding of their catalytic mechanisms are still required. It is well established that nitrogen dopants play a key role in boosting the activities of carbon materials in different catalytic reactions [25-30]. However, few efforts have been devoted to figuring out the relationship between different $\mathrm{N}$ species (pyridinic, pyrrolic, graphitic, and oxidized $\mathrm{N}$ ) 
and enzyme-like activities of N-CMNs, which retards further development of high-performance $\mathrm{N}$-CMNs and deeper understanding of structure-activity relationships of the catalyst at the atomic level. Due to the close formation energy of the $\mathrm{N}$ species, model nanozymes with well-controlled $\mathrm{N}$ dopants are still extremely challenging to obtain [31]. Towards this end, there is an urgent need to design model N-CMNs with modulated $\mathrm{N}$ dopants and identify the active sites of $\mathrm{N}$ CMNs, enabling an in-depth understanding of the nature of the catalysis and bringing unprecedented insights into the rational design of $\mathrm{N}-\mathrm{CMNs}$.

Herein, taking N-CMNs with well-controlled $\mathrm{N}$ dopants as model nanozymes, we present a successful paradigm to throw new light on the structure-activity relationship of $\mathrm{N}$ CMNs. By secondary $\mathrm{N}$ doping, $\mathrm{N}_{\mathrm{P}}-\mathrm{CMN}$ sere synthesized with porous structures and high pyridinic $\mathrm{N}$ content, which possessed superior POD-like activity. Unexpectedly, there was a positive correlation between the pyridinic $\mathrm{N}$ content and the intrinsic POD-like activity of N-CMNs. Theoretical calculations reveal that the pyridinic $\mathrm{N}$ dopant plays a key role in boosting the intrinsic POD-like activity of N-CMNs. Compared with graphitic and pyrrolic $\mathrm{N}$, pyridinic $\mathrm{N}$ can significantly reduce the endothermic reaction energy of the first $\mathrm{H}_{2} \mathrm{O}$ desorption step which is the key elementary step of the catalytic process. On this basis, $\mathrm{N}_{\mathrm{P}}-\mathrm{CMNs}$ were used to develop a sensing platform devoting to the assessment of the total antioxidant capacity (TAC), which is a key factor to evaluate the oxidative stress in healthcare and the food quality [32].

\section{Results and Discussion}

Using silica colloid as hard templates, N-CMNs-1 were synthesized via a pyrolysis process in the presence of glucosamine, targeting the simultaneous formation of porous structures and dispersed $\mathrm{N}$ dopants (Figure 1(a)). After secondary annealing treatment, $\mathrm{N}_{\mathrm{P}}$-CMNs were obtained using dicyandiamide as secondary nitrogen sources. As a control, $\mathrm{N}$-CMNs-2 were synthesized in the same manner without nitrogen sources. Given that dicyandiamide is decomposed to ammonia $\left(\mathrm{NH}_{3}\right)$ gas during annealing, the released $\mathrm{NH}_{3}$ can benefit the transformation of $\mathrm{N}$ species, achieving the modulation of nitrogen doping $[33,34]$. The transmission electron microscopy (TEM) image of the as-prepared $\mathrm{N}_{\mathrm{P}^{-}}$ CMNs is shown in Figure 1(b), which clearly displays the chiseled spherical pores with a diameter of $20 \mathrm{~nm}$, deriving from the silica templates. The ring-like selected area electron diffraction (SAED) pattern (Figure 1(b), inset) illustrates the amorphous nature of $\mathrm{N}_{\mathrm{P}}$-CMNs. Besides, as can be seen from the high-resolution TEM (HRTEM) image in Figure 1(c), $\mathrm{N}_{\mathrm{P}}$-CMNs consist of the curved graphene layers with a low crystallinity deduced from the unclear lattice fringes of graphitic carbon, which is consistent with the SAED result. As displayed in Figure S1, the introduction of secondary nitrogen sources makes no difference to the morphology of $\mathrm{N}-\mathrm{CMNs}$. High-angle annular dark-field scanning TEM (HADDF-STEM) image and the corresponding energy disperse spectroscopy (EDS) mappings (Figure 1(d)) demonstrate the homogeneous distribution of $\mathrm{C}, \mathrm{N}$, and $\mathrm{O}$ elements in the nanozymes. As shown in Figure 1(e), all the nanozymes exhibit obvious hysteresis loops in $\mathrm{N}_{2}$ adsorptiondesorption isotherms, indicating their mesoporous properties ascribed to the silica template packing. The BrunauerEmmett-Teller (BET) surface areas are 322.3, 309.2, and $302.5 \mathrm{~m}^{2} \mathrm{~g}^{-1}$ for $\mathrm{N}-\mathrm{CMNs}-1$, N-CMNs-2, and $\mathrm{N}_{\mathrm{P}}$-CMNs, respectively, indicating that the second pyrolysis has no obvious effect on their morphologies and surface areas. Figure 1(f) shows that the pore width of the nanozymes ranges from 2 to $30 \mathrm{~nm}$ (mesopores), in which most pores range around $20 \mathrm{~nm}$ with a normal distribution resulting from the silica templates. The hierarchical mesopores of N-CMNs not only increases the density of exposed active sites but also renders a faster mass transport, which is beneficial for further enhancing the nanozyme activities.

$\mathrm{X}$-ray powder diffraction (XRD) was investigated to assess the graphitization degree of the as-prepared $\mathrm{N}$ CMNs. As shown in Figure 2(a), two broad diffraction peaks of $\mathrm{N}-\mathrm{CMNs}$ centered at $2 \theta \approx 30$ and $42^{\circ}$ can be indexed to carbon (002) and (101), respectively, demonstrating the poor crystalline structure of the nanozymes $[9,35]$. Moreover, the nanozymes display the main Raman features of the $\mathrm{D}$ band at $1310 \mathrm{~cm}^{-1}$ and $\mathrm{G}$ band at $1590 \mathrm{~cm}^{-1}$ (Figure 2(b)). The peak intensity ratios of the $\mathrm{D}$ and $\mathrm{G}$ bands $\left(\mathrm{I}_{\mathrm{D}} / \mathrm{I}_{\mathrm{G}}\right)$ of the nanozymes are 0.92 (N-CMNs-1), 0.94 (N-CMNs-2), and 0.90 ( $\mathrm{N}_{\mathrm{P}}$-CMNs), respectively, suggesting all of them possess the similar defect density resulting from the doping of $\mathrm{N}$ atoms into the graphene network [36]. Together, in spite of the different pyrolysis procedures, no significant changes in the morphology and the crystalline structures of N-CMNs were observed from the various characterizations above. Then, Xray photoelectron spectroscopy (XPS) analyses were performed to identify the elemental compositions of N-CMNs, confirming the existence of $\mathrm{C}, \mathrm{N}$, and $\mathrm{O}$ (Figure S2). NCMNs synthesized with different $\mathrm{N}$ doping levels offer ideal models to investigate the role of $\mathrm{N}$ dopants in the catalytic process. As shown in Table S1, all N-CMNs possess high atom ratios of total nitrogen (5.85-6.21\%), which is higher than those of some reported $\mathrm{N}$-doped carbons [10, 30, 35]. The configuration of the doped $\mathrm{N}$ species was further determined by deconvolution $\mathrm{N}$ 1s spectra (Figure 2(c)). There are four typical peaks at 398.33, 399.35, 401.15, and $402.33 \mathrm{eV}$, assigned to pyridinic, pyrrolic, graphitic, and oxidized $\mathrm{N}$, respectively. It is found that graphitic $\mathrm{N}$ is the predominant peak in $\mathrm{N}$ 1s XPS spectra, while pyrrolic, graphitic, and oxidized $\mathrm{N}$ for the three types of $\mathrm{N}-\mathrm{CMNs}$ were observed with similar content, respectively (Figure 2(d) and Table S2). Note that the atomic percent of pyridinic $\mathrm{N}$ of $\mathrm{N}_{\mathrm{P}}-\mathrm{CMNs}$ is $1.02 \%$, remarkably higher than those of $\mathrm{N}$ CMNs-1 (0.828\%) and N-CMNs-2 (0.832\%), which is expected to exert great influence in the nanozyme activities.

The POD-like activity of N-CMNs was investigated and contrasted by monitoring 3, 3', 5, 5' -tetramethylbenzidine (TMB) oxidation taking hydrogen peroxide $\left(\mathrm{H}_{2} \mathrm{O}_{2}\right)$ as substrate. As shown in Figure 3(a), the solution containing $\mathrm{N}_{\mathrm{P}^{-}}$ CMNs shows a much higher absorbance of oxidized TMB (oxTMB) at $652 \mathrm{~nm}$ than those of the solution containing N-CMNs-1 and N-CMNs-2, indicating that the POD-like activity of $\mathrm{N}_{\mathrm{P}}$-CMNs is remarkably enhanced after secondary 


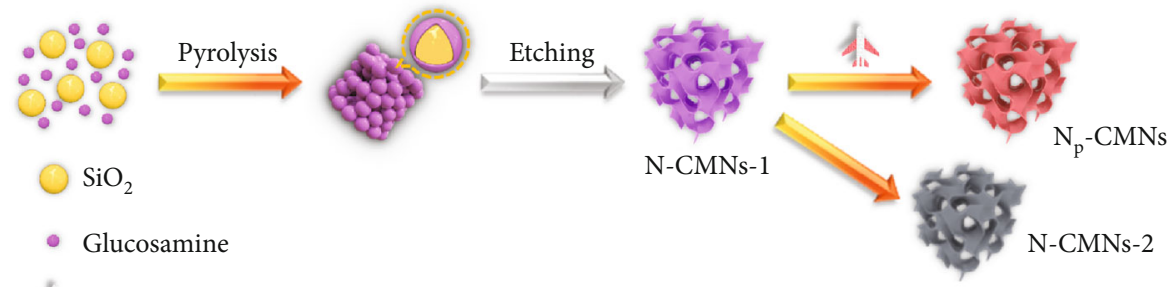

(a)

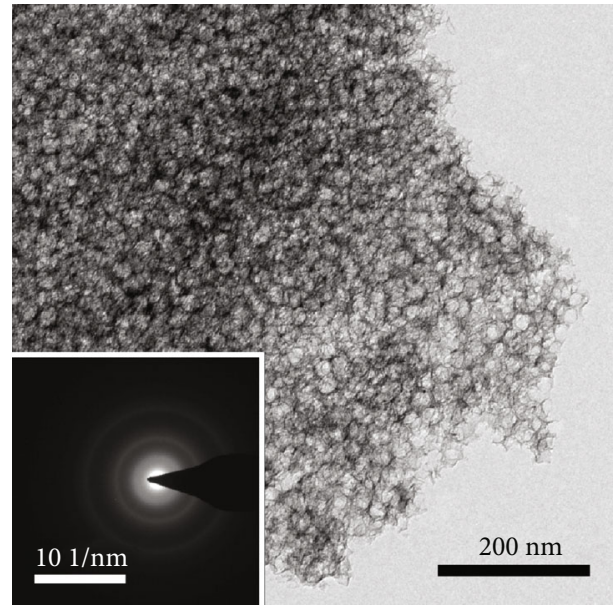

(b)

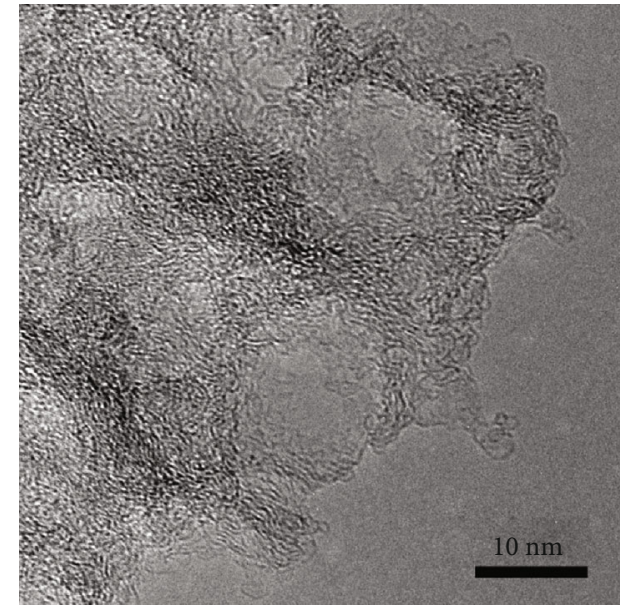

(c)
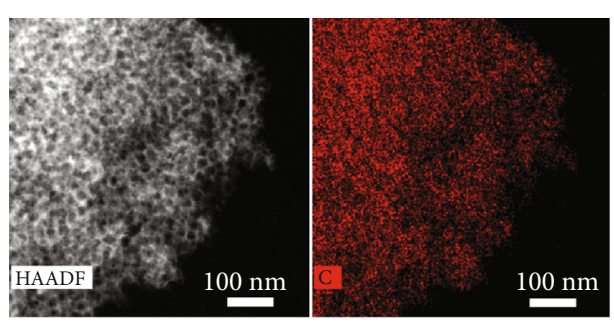

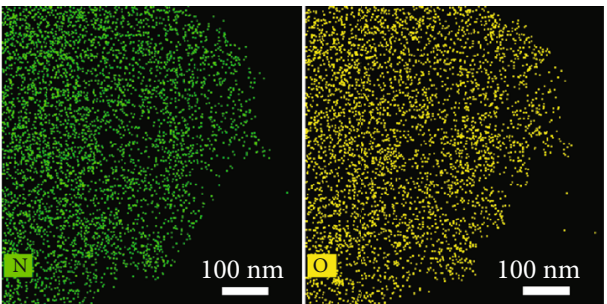

(d)

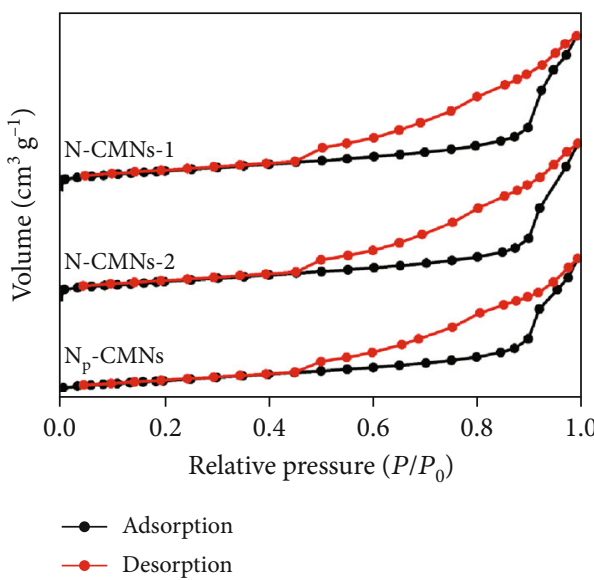

(e)

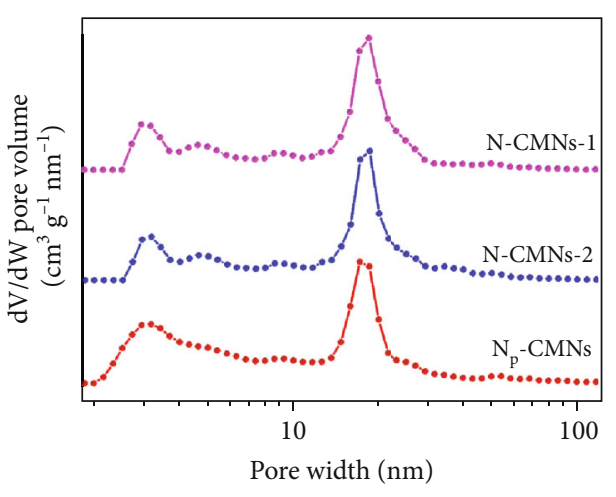

(f)

FIGURE 1: (a) Schematic illustration of the synthesis of N-CMNs. TEM image (b), SAED pattern (b, inset), HRTEM image (c), and HAADFSTEM image and the corresponding EDS mappings (d) of $\mathrm{N}_{\mathrm{P}}$-CMNs. $\mathrm{N}_{2}$ physisorption isotherm (e) and pore width distribution (f) of different N-CMNs. 

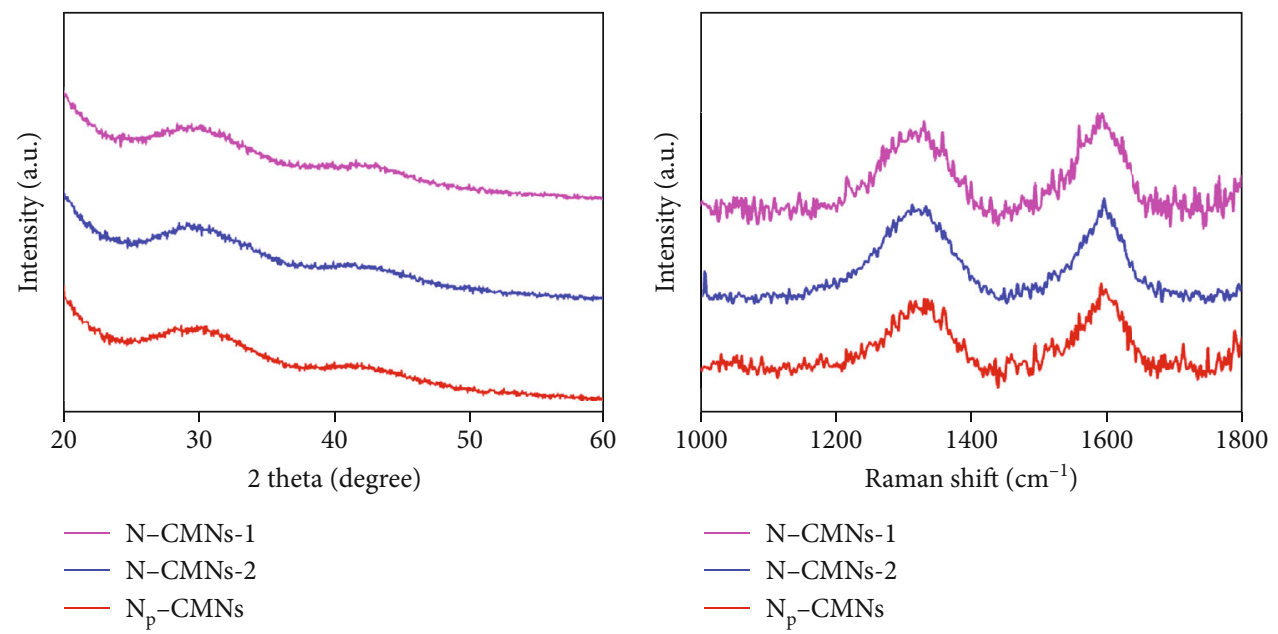

(a)

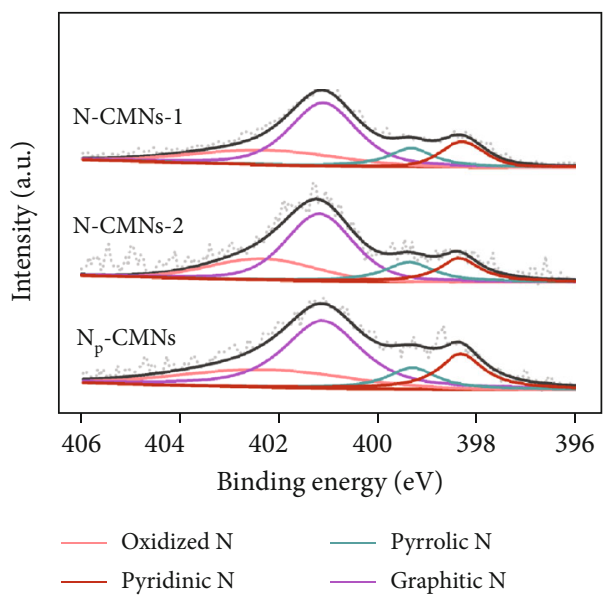

(b)

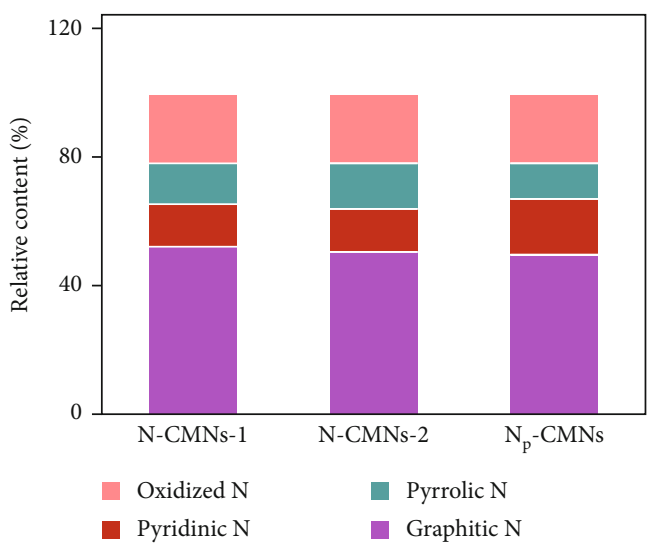

(c)

(d)

FIgURE 2: XRD patterns (a) and Raman spectra (b) of N-CMNs-1, N-CMNs-2, and $\mathrm{N}_{\mathrm{P}}$-CMNs. N 1s high-resolution XPS spectra (c) and the corresponding relative contents of different $\mathrm{N}$ species (d) of the as-prepared nanozymes.

$\mathrm{N}$-doping. As expected, the catalytic rates of the nanozymecatalyzed reaction increase gradually and reach a plateau with the increase of substrate concentration (Figure S3), which suggests that the $\mathrm{N}$-CMNs-catalyzed reactions obey the Michaelis-Menten kinetics. As shown in Figure 3(b) and Table S3, $\mathrm{N}_{\mathrm{P}}-\mathrm{CMNs}$ exhibit the highest POD-like activity according to the values of the Michaelis-Menten constant $\left(K_{M}\right)$ and maximal velocity $\left(V_{\max }\right)$, which are the key kinetic parameters for estimating the performance of nanozymes [37]. Meanwhile, the proposed $\mathrm{N}_{\mathrm{P}}-\mathrm{CMNs}$ have competitive POD-like activities compared with other reported nanozymes (Table S4) [38-42]. In addition, the specific activity (SA), defined as activity unit (U) per milligram (mg) of nanozyme, was quantitatively contrasted to further investigate the POD-like activities of the $\mathrm{N}$ CMNs (Figure 3(c)). The SA of $\mathrm{N}_{\mathrm{P}}-\mathrm{CMNs}$ is $16.2 \mathrm{U} \mathrm{mg}^{-1}$, much higher than those of N-CMNs-1 $\left(9.1 \mathrm{U} \mathrm{mg}^{-1}\right)$ and $\mathrm{N}-$ CMNs-2 (11.5 $\left.\mathrm{U} \mathrm{mg}^{-1}\right)$, indicating the superior activity of $\mathrm{N}_{\mathrm{P}}$-CMNs. As can be seen from Figure $3(\mathrm{~d})$, there is an unexpected positive correlation between the relative content of pyridinic $\mathrm{N}$ and the SA of $\mathrm{N}-\mathrm{CMNs}$, which suggests that the pyridinic $\mathrm{N}$ dopant plays a crucial role in improving the POD-like activities of N-CMNs. The optimized condition for the POD-like catalysis of $\mathrm{N}_{\mathrm{P}}-\mathrm{CMNs}$, including $\mathrm{pH}$ and temperature, were investigated (Figure S4). The activity of $\mathrm{N}_{\mathrm{P}}-\mathrm{CMNs}$ reaches its maximum at $\mathrm{pH} 3.0$, and $\mathrm{N}_{\mathrm{P}}$-CMNs possess a high tolerance to the change of temperature in comparison with natural horseradish peroxidases (HRP).

To further elucidate the active sites and catalytic mechanism, POD-like activities on the three types of $\mathrm{N}$-doped model graphene sheets (graphitic N, pyridinic N, and pyrrolic $\mathrm{N}$ models) were investigated by density functional theory (DFT) calculations. Notably, it is generally accepted that hydroxyl radicals $(\bullet \mathrm{OH})$ are the active intermediates of the catalytic reactions involving carbon nanozymes [11]. Unexpectedly, as displayed in the electron spin resonance (ESR) spectra (Figure S5a), there is no $\bullet \mathrm{OH}$ generated and captured during the catalytic process of $\mathrm{N}$-CMNs. In addition, the p-phthalic acid (PTA) is used as a fluorescence probe of $\bullet \mathrm{OH}$ while no characteristic emission of the fluorescent product near $430 \mathrm{~nm}$ is observed in Figure S5b, agreed well with the abovementioned result 

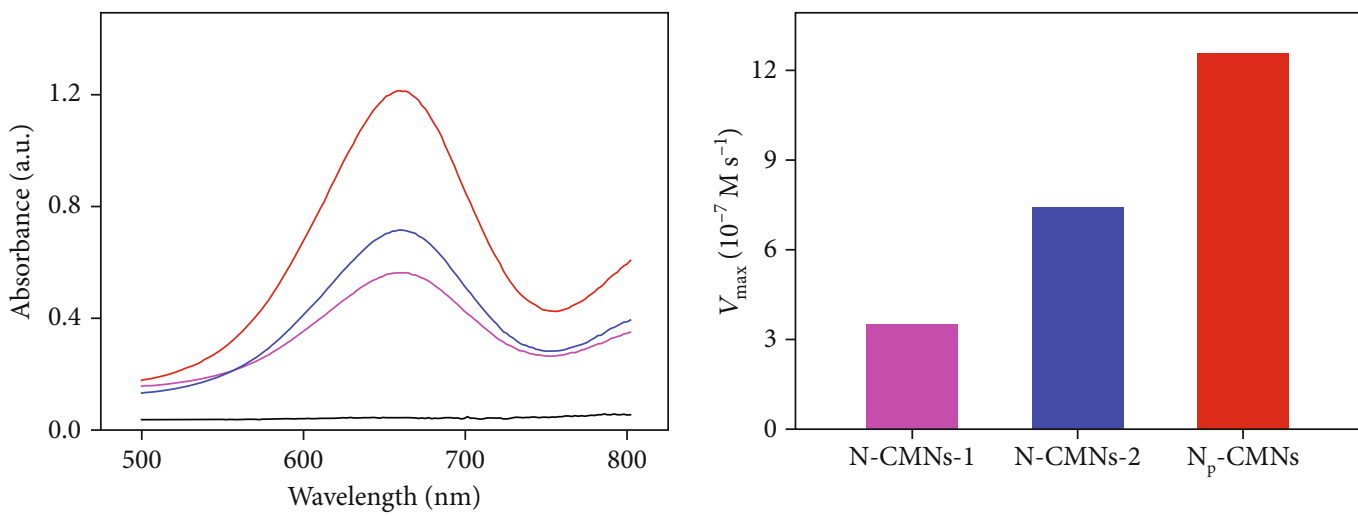

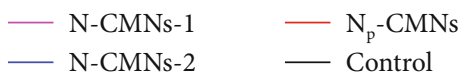

(a)

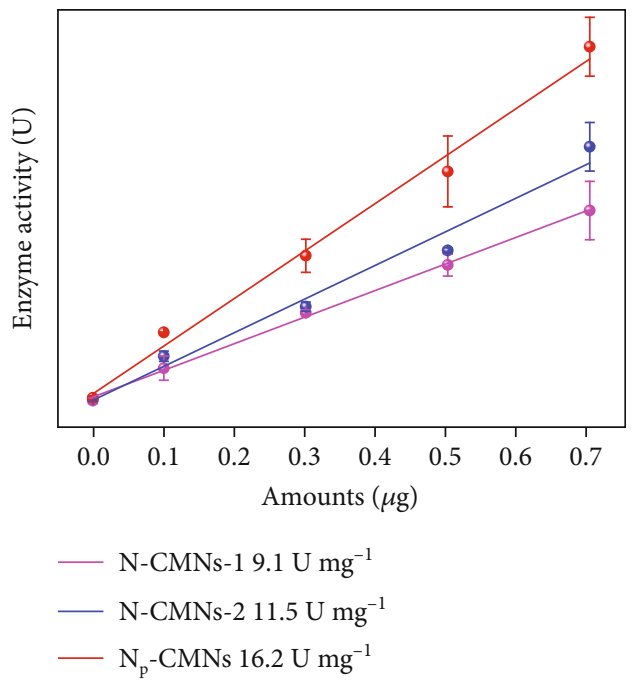

(c) (b)

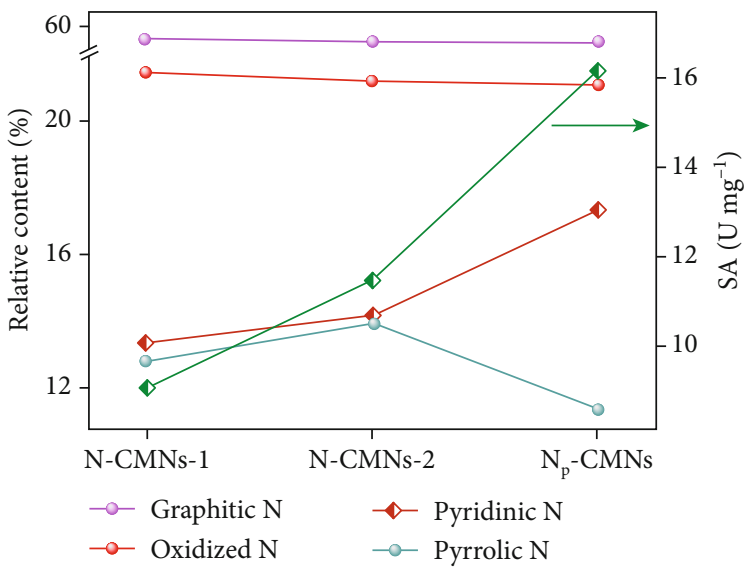

FIGURE 3: (a) UV-vis absorption spectra of POD-like catalytic oxidation in the presence of N-CMNs. Comparison of the $V_{\max }$ (b) (taking $\mathrm{H}_{2} \mathrm{O}_{2}$ as substrate) and the SA (c) of N-CMNs. (d) The correlation between the relative content of N dopants and the SA of N-CMNs.

from the ESR spectra. In consequence, we speculate that the other high oxidation-state intermediates dominate the PODlike catalysis of N-CMNs, which is similar to the natural HRP [43]. Then, the catalytic process of N-CMNs is considered to proceed as the following six elementary steps [12]:

$$
\begin{gathered}
\mathrm{H}_{2} \mathrm{O}_{2}+* \rightarrow \mathrm{H}_{2} \mathrm{O}_{2}{ }^{*} \\
\mathrm{H}_{2} \mathrm{O}_{2}{ }^{*}+* \rightarrow \mathrm{H}_{2} \mathrm{O}^{*}+\mathrm{O}^{*} \\
\mathrm{H}_{2} \mathrm{O}^{*}+\mathrm{O}^{*} \rightarrow \mathrm{O}^{*}+\mathrm{H}_{2} \mathrm{O}+* \\
\mathrm{O}^{*}+\mathrm{H}^{+}+\mathrm{e}^{-} \rightarrow \mathrm{HO}^{*} \\
\mathrm{HO}^{*}+\mathrm{H}^{+}+\mathrm{e}^{-} \rightarrow \mathrm{H}_{2} \mathrm{O}^{*} \\
\mathrm{H}_{2} \mathrm{O}^{*} \rightarrow \mathrm{H}_{2} \mathrm{O}+*
\end{gathered}
$$

where the asterisk indicates the adsorption site. Steps 1-3 are considered as the $\mathrm{H}_{2} \mathrm{O}_{2}$ decomposition steps, while steps 4 and 5 are the proton-electron pair transfer steps. As shown in Figure 4(a), taking the carbon atoms connected to $\mathrm{N}$ as the active sites, $\mathrm{H}_{2} \mathrm{O}_{2}$ is first adsorbed on $\mathrm{N}$-doped model graphene, and then, a deoxidation process of $\mathrm{H}_{2} \mathrm{O}_{2}$ occurs, followed by the desorption of a $\mathrm{H}_{2} \mathrm{O}$ molecule and the formation of oxidation-state intermediate 4 in which the $\mathrm{O}$ atom is connected to the active site on the top site of graphitic $\mathrm{N}$ model and bridge site of pyridinic $\mathrm{N}$ and pyrrolic $\mathrm{N}$ models, respectively. On the premise of providing protons by substrate TMB, the two proton-electron pair transfer steps are sequentially achieved, followed by the formation and removal of $\mathrm{H}_{2} \mathrm{O}$. Based on the above reaction path, the potential energy diagram was obtained in Figure 4(b), where steps 3 and 6 were found to be the only two endothermic steps. The calculation results show that step 6 on the three models have similar reaction energies of about $0.36 \mathrm{eV}$, which demonstrates 


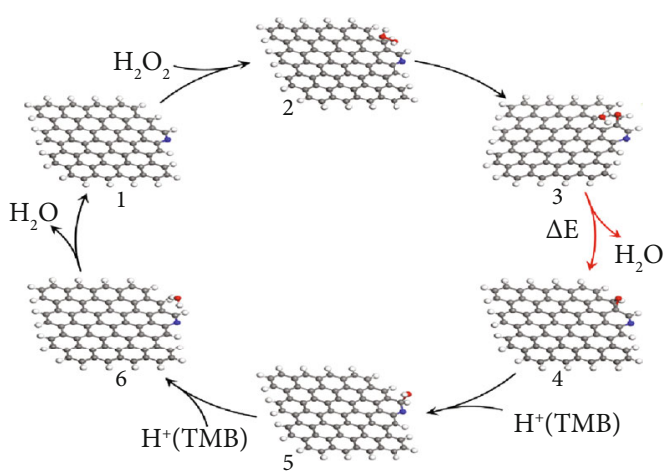

(a)
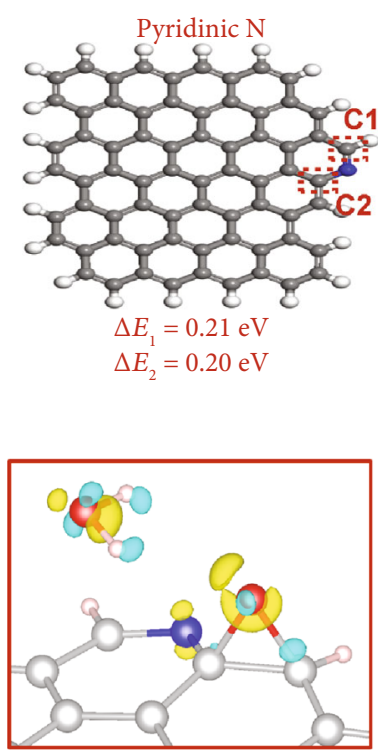

(c)

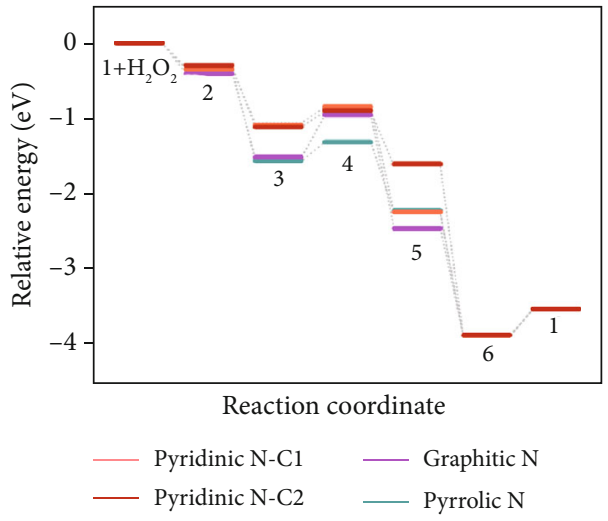

(b)
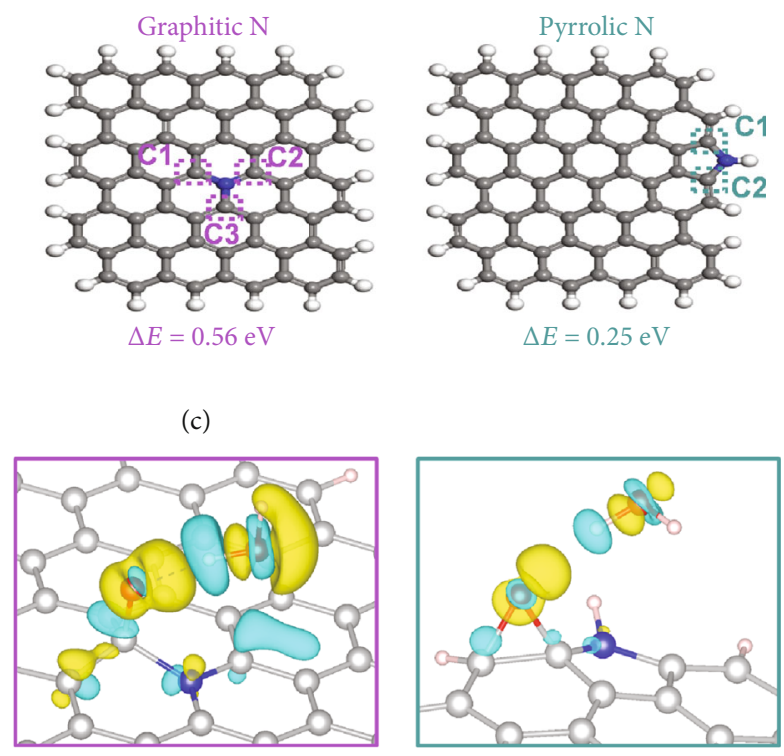

(d)

FIgURE 4: (a) The process of POD-like catalytic reaction on pyridinic N-doped model graphene. (b) Energy profile for POD-like reactions on three types of $\mathrm{N}$-doped model graphene sheets. (c) The optimized structures of graphitic N, pyridinic $\mathrm{N}$, and pyrrolic $\mathrm{N}$ models and the corresponding reaction energies of the key endothermic step 3. (d) Difference charge densities of $\mathrm{H}_{2} \mathrm{O}$ adsorption on the oxidation-state intermediate 3 (isosurface value is $0.008 \mathrm{e}^{-3}$ ). The blue and yellow regions indicate the depletion and the accumulation of electronic charge, respectively.

that $\mathrm{H}_{2} \mathrm{O}$ desorption in step 6 has a negligible difference among the three $\mathrm{N}$-doped model graphene in the perspective of thermodynamics. Thus, step 3 is considered as the key endothermic step in the catalytic process, which is responsible for the catalytic performances of N-CMNs. As shown in Figure 4(c), the reaction energies of step 3 on the graphitic $\mathrm{N}$ model and pyrrolic $\mathrm{N}$ model are $0.56 \mathrm{eV}$ and $0.25 \mathrm{eV}$, respectively, higher than those of $\mathrm{C} 1(0.21 \mathrm{eV})$ and $\mathrm{C} 2$ $(0.20 \mathrm{eV})$ on the pyridinic $\mathrm{N}$ model, indicating that the doping of pyridinic $\mathrm{N}$ significantly reduces the endothermic reaction energy of step 3 and boosts the intrinsic POD-like activities of $\mathrm{N}-\mathrm{CMNs}$. To further explain the influence of different $\mathrm{N}$ doped species on the reaction activity of step 3 , the electronic structural properties of three models and their reaction intermediates were investigated. As shown in Table S5, the Bader charges of structure 1 and $\mathrm{H}_{2} \mathrm{O}$-adsorbed oxidationstate intermediate 3 were determined. In comparison with other $\mathrm{N}$ species, the active site $\mathrm{C}$ atom adjacent to pyridinic $\mathrm{N}$ has a more positive charge. Besides, the $\mathrm{O}$ atom connected to the active site $\mathrm{C}$ atom in the intermediate 3 of the pyridinic $\mathrm{N}$ model shows less negative charge than that of graphitic and pyrrolic $\mathrm{N}$ models, which gives rise to weaker adsorption of $\mathrm{H}_{2} \mathrm{O}$ in intermediate 3 and thus easier desorption of $\mathrm{H}_{2} \mathrm{O}$ during the key endothermic step 3. In addition, as displayed in Figure 4(d), the corresponding difference charge densities of three models further confirm the charge transfer between adsorbed $\mathrm{H}_{2} \mathrm{O}$ and active site in intermediate 3, which also suggests the less negative charge of $\mathrm{O}$ atom in pyridinic $\mathrm{N}$ model and the weaker interaction between positively charged $\mathrm{H}$ atoms in adsorbed $\mathrm{H}_{2} \mathrm{O}$ and $\mathrm{O}$ atom. Taken together, pyridinic $\mathrm{N}$ dopant is conducive for the generated $\mathrm{H}_{2} \mathrm{O}$ to desorb during the key endothermic step, eventually of great benefit to boost the POD-like activities of N-CMNs.

TAC is a vital index to assess the quality of medicine and antioxidant food for healthcare [32], which can be identified by detecting the millimolar equivalent of ascorbic acid (AA) 


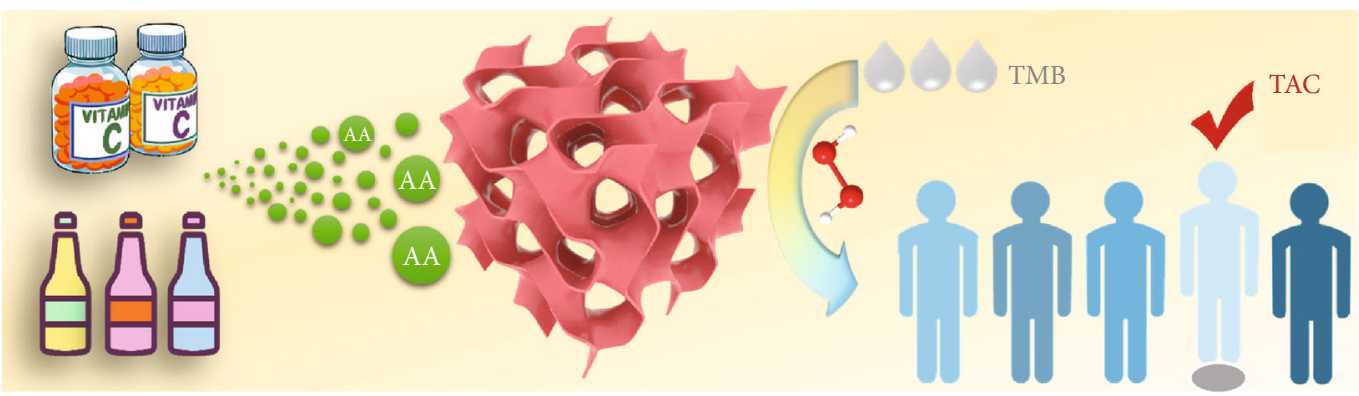

(a)
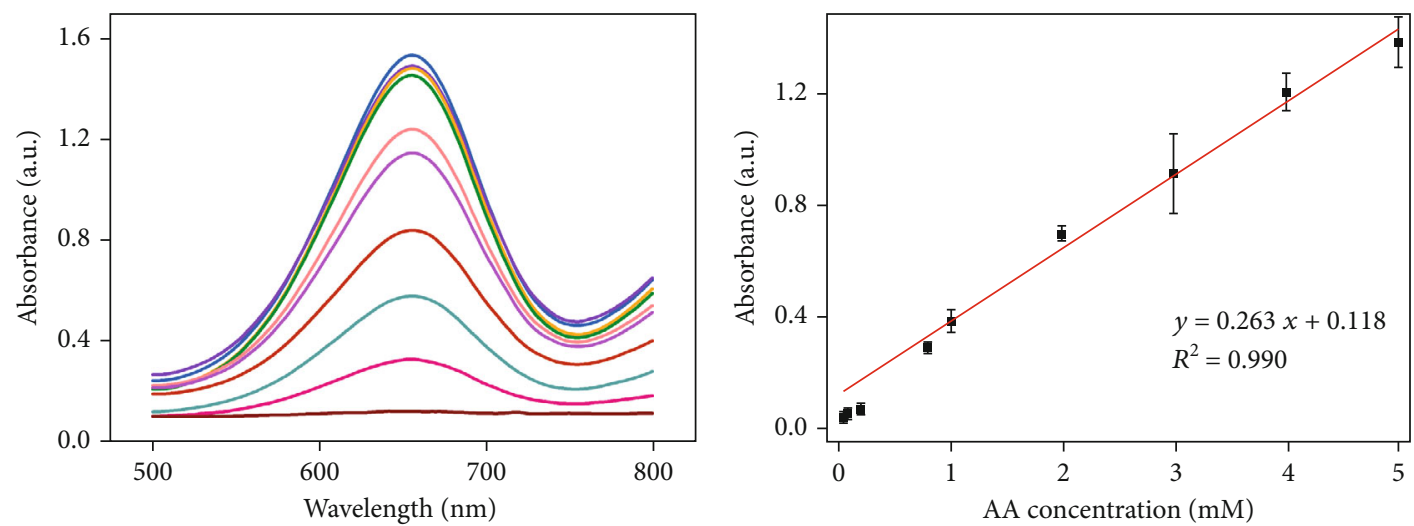

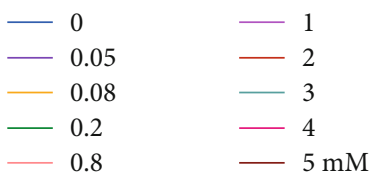

(b)

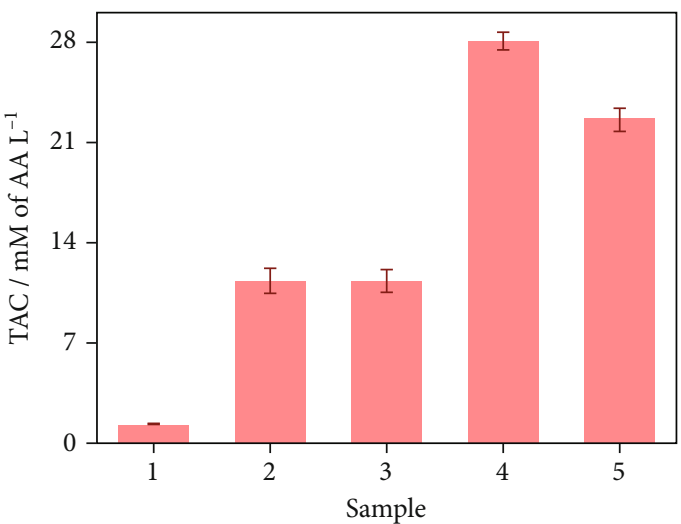

(d)

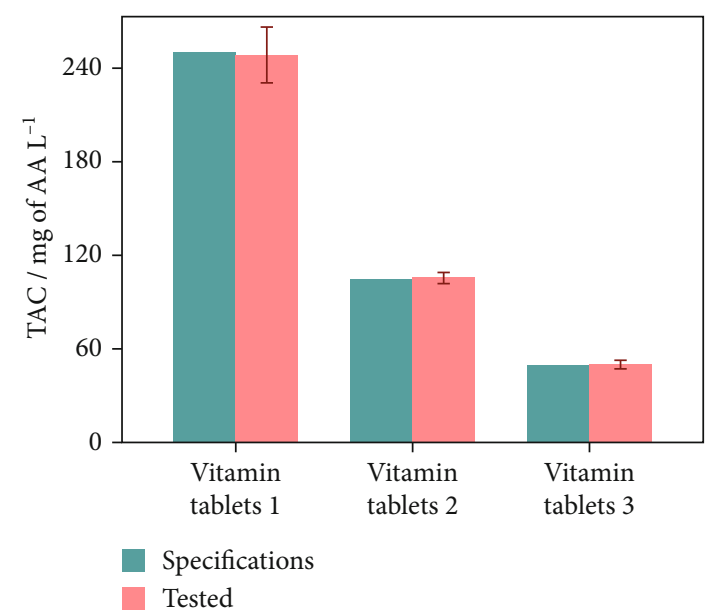

(e)

FIgURE 5: (a) Schematic illustration of the TAC assay using $\mathrm{N}_{\mathrm{P}}$-CMNs. UV-vis spectra (b) and corresponding calibration curves (c) of the TAC assay with the AA concentrations from 0.05 to $5 \mathrm{mM}$. TAC of commercial beverages (d) and vitamin tablets (f) (in comparison with the specifications).

$\mathrm{L}^{-1}$. Taking advantage of the superior POD-like activity, $\mathrm{N}_{\mathrm{P}^{-}}$ CMNs are beneficial for implementing TAC assay as a concept application (Figure 5(a)). AA, also known as vitamin $\mathrm{C}$, is a common water-soluble micronutrient applied in the prevention and treatment of disease and health care [44, 45]. Given that AA is a strong reducing agent, it can effi- ciently reduce oxTMB to TMB and consume $\mathrm{H}_{2} \mathrm{O}_{2}$, leading to a decrease in the absorbance of oxTMB [46]. Based on this suggested mechanism, a series of AA concentrations could be monitored (Figure 5(b)), which demonstrated the feasibility of the TAC assay. As shown in Figure S6, the optimized conditions of $\mathrm{pH} 3.0$ and nanozyme amount of $10 \mu \mathrm{L}$ were 
adopted for the detection of AA. Accordingly, as displayed in Figure $5(\mathrm{c})$, a clear linear relationship $\left(R^{2}=0.99\right)$ was determined between the absorbance at $652 \mathrm{~nm}$ and the concentration of AA ranging from 0.05 to $5 \mathrm{mM}$, with a limit of detection (LOD) of $0.03 \mathrm{mM}(\mathrm{S} / \mathrm{N}=3)$. To further develop the practical use of the assay, the TAC of five commercial beverages (Figure 5(d)) and three vitamin tablets (Figure 5(e)) were detected. The results closely coincide with the specifications from the samples, indicating that the samples meet the quality requirements. Moreover, the potential interferents in food and medicine did not affect the detection (Figure S7), which revealed the robustness of the assay.

\section{Conclusion}

In summary, utilizing the secondary nitrogen doping, we synthesized porous $\mathrm{N}_{\mathrm{P}}-\mathrm{CMN}$ with high pyridinic $\mathrm{N}$ content and superior POD-like activity. There is an unexpected finding that the intrinsic POD-like activity of N-CMNs can be modulated by varying the pyridinic $\mathrm{N}$ content, revealing that the specific active sites of $\mathrm{N}$-CMNs are relative to the pyridinic $\mathrm{N}$ dopant. The mechanism study demonstrated that the pyridinic $\mathrm{N}$ dopant renders the active site region less negatively charged in $\mathrm{H}_{2} \mathrm{O}$-adsorped oxidation-state intermediate in comparison with other $\mathrm{N}$ species, which is beneficial to reduce the first $\mathrm{H}_{2} \mathrm{O}$ desorption energy in thermodynamics and boost the intrinsic POD-like activities of N-CMNs. Moreover, the PODlike property of $\mathrm{N}$-CMNs allows them to serve as biocatalysts for bioapplications. Taking advantage of the superior PODlike activity of $\mathrm{N}_{\mathrm{P}}$-CMNs, a TAC assay was successfully developed, which holds great promise in conducting a quality evaluation of medicine tablets and antioxidant food.

\section{Materials and Methods}

4.1. Chemicals and Materials. 5, 5-Dimethyl-1-pyrroline-Noxide, PTA, and TMB were purchased from Aladdin Chemical Reagent Co., Ltd. (Shanghai, China). Glucosamine hydrochloride, dicyandiamide, hydrofluoric acid (HF), acetic acid (HAc), sodium acetate $(\mathrm{NaAc})$, hydrogen peroxide $(30 \%)$, and AA were obtained from Sinopharm Chemical Reagent Co., Ltd. (Shanghai, China). Colloidal silica 40 wt.\% suspension $\left(\mathrm{SiO}_{2}\right)$ was purchased from Sigma-Aldrich. All chemicals were of analytical grade, while solutions were prepared with deionized water (18.2 $\mathrm{M} \Omega \mathrm{cm}$, Millipore).

4.2. Instrumentation. The TEM images were carried out on a Titan Themis G2 60-300 (Thermo Fisher, United States). Nitrogen physisorption isotherms were measured on a TriStar II 3 flex instrument. XRD patterns were obtained utilizing a D8 ADVANCE (Bruker, Germany). XPS was performed by Thermo ESCALAB 250XI (Thermo Fisher, United States) with an X-ray source $(\mathrm{Mg} \mathrm{K} \alpha \mathrm{h} v=1486.6 \mathrm{eV})$. Raman spectra were measured by a Renishaw inVia Raman spectrometer (Thermo Fisher DXRxi). All the UV-vis absorption spectra were performed by a microplate reader (Tecan Spark, Switzerland). The ESR spectra were obtained from an ESR spectrometer (Bruker EMX plus, Germany).
4.3. Synthesis of $\mathrm{N}$-CMNs. Typically, $5 \mathrm{~g}$ of glucosamine hydrochloride was dissolved in $20 \mathrm{~mL}$ of colloidal silica suspension $\left(2 \mathrm{~g} \mathrm{SiO}_{2}\right)$. After stirred for $30 \mathrm{~min}$, the derived solution was then freeze-dried. The obtained powder was annealed under flowing $\mathrm{N}_{2}$ with a heating rate of $5{ }^{\circ} \mathrm{C} \mathrm{min}^{-1}$ to $900{ }^{\circ} \mathrm{C}$, and the temperature was kept for $2 \mathrm{~h}$. After that, hierarchically porous $\mathrm{N}-\mathrm{CMNs}-1$ were obtained after etching for $12 \mathrm{~h}$ by $\mathrm{HF}$ ( 10 wt.\%) which can remove $\mathrm{SiO}_{2}$ templates, followed by drying at $60^{\circ} \mathrm{C}$ for $12 \mathrm{~h}$. $\mathrm{N}_{\mathrm{P}}$-CMNs were obtained taking dicyandiamide as secondary nitrogen sources. In brief, $500 \mathrm{mg}$ of N-CMNs-1 was mixed with $1 \mathrm{~g}$ of dicyandiamide by grounding, and the mixture was heated to $900{ }^{\circ} \mathrm{C}$ with a heating rate of $3{ }^{\circ} \mathrm{C} \mathrm{min}^{-1}$. After keeping at $900{ }^{\circ} \mathrm{C}$ for $1 \mathrm{~h}$, $\mathrm{N}_{\mathrm{P}}$-CMNs were procured directly. For comparison, NCMNs-2 were synthesized in the absence of dicyandiamide.

4.4. Measurement of the POD-Like Activity of N-CMNs. The POD-like activity of $\mathrm{N}-\mathrm{CMNs}$ was measured taking TMB and $\mathrm{H}_{2} \mathrm{O}_{2}$ as substrates. Typically, the steady-state kinetic assays were implemented in HAc-NaAc buffer $(0.1 \mathrm{M}$, $\mathrm{pH}$ 3.0) containing N-CMN solution $\left(5 \mu \mathrm{L}, 1 \mathrm{mg} \mathrm{mL}^{-1}\right)$ as catalysts. The kinetic assays of N-CMNs taking TMB as substrate were conducted by adding $\mathrm{H}_{2} \mathrm{O}_{2}(100 \mathrm{mM}, 100 \mu \mathrm{L})$ and $\mathrm{TMB}$ with different concentrations $(1,1.5,2,3,4,5,7 \mathrm{mM}$, $100 \mu \mathrm{L}$ ), while the kinetic assays of $\mathrm{N}$-CMNs taking $\mathrm{H}_{2} \mathrm{O}_{2}$ as substrate were carried out by adding TMB $(10 \mathrm{mM}$, $100 \mu \mathrm{L})$ and $\mathrm{H}_{2} \mathrm{O}_{2}$ with different concentrations $(20,25,30$, $40,50,60,70 \mu \mathrm{M}, 100 \mu \mathrm{L})$. Then, the absorbance of oxTMB at $652 \mathrm{~nm}$ was measured at a regular interval. Subsequently, $K_{M}$ and $V_{\max }$ were determined by the Michaelis-Menten curve.

4.5. DFT Calculations. The catalytic mechanism was studied by DFT calculations which were performed using the Vienna $\mathrm{Ab}$ initio Simulation Package (VASP) [47, 48]. The interactions between ion cores and electrons were described employing the Projector Augmented Wave (PAW) [49], and the exchange-correlation energy was computed using the Perdew, Burke, and Ernzerhof functional (PBE) [50]. The plane wave basis sets with a cutoff energy of $400 \mathrm{eV}$ were used. The Brillouin zone sampling was restricted to the $\Gamma$ point with $\mathrm{K}$-point meshes $1 \times 1 \times 1$. The conjugate gradient method was used for geometry optimizations, and the maximum forces on all atoms were smaller than $0.05 \mathrm{eV}^{-1}$, while the energy convergence criterion of the electronic step was set to $10^{-4} \mathrm{eV}$. The structures of all reactant complex, reaction intermediate, and product complex that exist in POD-like catalytic reaction on $\mathrm{N}$-doped model graphene were optimized with lattice constants of $a=23.00 \AA, b=11.00 \AA$, and $c=26.00 \AA$. All atoms were allowed to relax. Bader charge analysis was performed to investigate the electronic structural properties [51].

The $\mathrm{H}_{2} \mathrm{O}$ desorption energies $(\Delta \mathrm{E})$ on intermediate 3 were calculated by:

$$
\Delta E=E_{(\mathrm{oxi})}+E_{\left(\mathrm{H}_{2} \mathrm{O}\right)}-E_{\left(\mathrm{oxi}+\mathrm{H}_{2} \mathrm{O}\right)},
$$

where $E_{(\text {oxi })}, E_{\left(\mathrm{H}_{2} \mathrm{O}\right)}$, and $E_{\left(\text {oxi }+\mathrm{H}_{2} \mathrm{O}\right)}$ are the energies of 
intermediate $4, \mathrm{H}_{2} \mathrm{O}$ molecule in the gas phase, and $\mathrm{H}_{2} \mathrm{O}$ adsorbed intermediate 3 .

The difference charge density of the three models was defined as:

$$
\Delta \rho=\rho_{\text {int }-3}-\rho_{\text {oxi }}-\rho_{\mathrm{H}_{2} \mathrm{O}},
$$

where $\rho_{\text {int-3 }}, \rho_{\text {oxi }}$, and $\rho_{\mathrm{H}_{2} \mathrm{O}}$ stand for the charge densities of intermediate 3 , oxidation-state intermediate without $\mathrm{H}_{2} \mathrm{O}$ adsorption, and adsorbed $\mathrm{H}_{2} \mathrm{O}$ molecule, respectively.

4.6. TAC Assay. In a typical procedure, $\mathrm{N}_{\mathrm{P}}$-CMNs $(10 \mu \mathrm{L}$, $\left.1 \mathrm{mg} \mathrm{mL}^{-1}\right), \mathrm{HAc}-\mathrm{NaAc}$ buffer $(150 \mu \mathrm{L} 0.1 \mathrm{M}, \mathrm{pH} 3.0), \mathrm{H}_{2} \mathrm{O}_{2}$ $(50 \mu \mathrm{L}, 100 \mathrm{mM})$, TMB $(50 \mu \mathrm{L}, 5 \mathrm{mM})$, and $50 \mu \mathrm{L}$ of AA with a series of concentrations were incubated for $5 \mathrm{~min}$, followed by measuring the absorbance of oxTMB $(652 \mathrm{~nm})$ in the solution. The same protocol was carried out to determine the TAC of practical samples, including commercial beverages and vitamin tablets, after adjusting their concentration within the detection range. The selectivity of the assay was investigated by interference, including metal ions, carbohydrates, and amino acids. The concentration of AA was $4 \mathrm{mM}$, while the concentrations of the interferences were $100 \mathrm{mM}$.

\section{Conflicts of Interest}

The authors declare no competing financial interest.

\section{Authors' Contributions}

Hongye Yan and Linzhe Wang contributed equally to this work.

\section{Acknowledgments}

The authors gratefully acknowledge the financial support of the Fundamental Research Funds for the Central Universities (CCNU 20TS013), the National Natural Science Foundation of China (no. 21503273), and the Program of Introducing Talents of Discipline to Universities of China (111 programs, B17019).

\section{Supplementary Materials}

Figure S1. TEM images and HRTEM images (inset) of NCMNs-1 (a) and N-CMNs-2 (b). Figure S2. XPS spectra of $\mathrm{N}$-CMNs-1, N-CMNs-2 and $\mathrm{N}_{\mathrm{p}}$-CMNs. Figure S3. The steady-state kinetics of N-CMNs (a-d: N-CMNs-1, e-h: NCMNs-2, i-l: $\mathrm{N}_{\mathrm{P}}-\mathrm{CMNs}$ ) toward various concentrations of $\mathrm{H}_{2} \mathrm{O}_{2}$ and TMB, respectively. Figure S4. pH (a) and temperature (b)-dependent activity for $\mathrm{N}_{\mathrm{P}}$-CMNs and horseradish peroxidases. Figure S5. (a) ESR spectra of free radicals produced in the catalytic processes. (b) Fluorescence spectra of the solution with PTA, PTA $+\mathrm{H}_{2} \mathrm{O}_{2}, \mathrm{~N}_{\mathrm{P}}$-CMNs+PTA $+\mathrm{H}_{2} \mathrm{O}_{2}$. Figure $\mathrm{S} 6$. Absorbance of reaction systems in the absence and presence of ascorbic acid (AA) varied with $\mathrm{pH}$ (a) and nanozymes amount (b). Figure S7. Selective detection of TAC assay. Table S1. Comparison of carbon, oxygen and nitrogen contents [atom \%] in the studied N-CMNs in this work with others obtained from XPS. Table S2. Surface concentrations of nitrogen species obtained by fitting the $\mathrm{N} 1 \mathrm{~s}$ core-level XPS spectra. Table S3. The Michaelis-Menten constant $\left(\mathrm{K}_{\mathrm{M}}\right)$ and maximum reaction rate $\left(\mathrm{V}_{\max }\right)$ of the $\mathrm{N}$ CMNs for POD-like catalysis. Table S4. Comparison of the kinetic parameters of $\mathrm{N}-\mathrm{CMNs}$ and other reported nanozymes. Table S5. The Bader charge of atoms in the intermediate (Int) 1 and 3 of $\mathrm{N}$-doped model graphene. (Supplementary Materials)

\section{References}

[1] D. Jiang, D. Ni, Z. T. Rosenkrans, P. Huang, X. Yan, and W. Cai, "Nanozyme: new horizons for responsive biomedical applications," Chemical Society Reviews, vol. 48, no. 14, pp. 3683-3704, 2019.

[2] J. Wu, X. Wang, Q. Wang et al., "Nanomaterials with enzymelike characteristics (nanozymes): next-generation artificial enzymes (II)," Chemical Society Reviews, vol. 48, no. 4, pp. 1004-1076, 2019.

[3] H. Wang, K. Wan, and X. Shi, "Recent advances in nanozyme research," Advanced Materials, vol. 31, no. 45, article 1805368, 2018.

[4] Y. Huang, J. Ren, and X. Qu, "Nanozymes: classification, catalytic mechanisms, activity regulation, and applications," Chemical Reviews, vol. 119, no. 6, pp. 43574412, 2019.

[5] P. B. O'Mara, P. Wilde, T. M. Benedetti et al., "Cascade reactions in nanozymes: spatially separated active sites inside Agcore-porous-Cu-shell nanoparticles for multistep carbon dioxide reduction to higher organic molecules," Journal of the American Chemical Society, vol. 141, no. 36, pp. 1409314097, 2019.

[6] L. Jiao, H. Yan, Y. Wu et al., "When nanozymes meet singleatom catalysis," Angewandte Chemie International Edition, vol. 132, no. 7, pp. 2585-2596, 2020.

[7] Q. Wang, H. Wei, Z. Zhang, E. Wang, and S. Dong, "Nanozyme: an emerging alternative to natural enzyme for biosensing and immunoassay," TrAC Trends in Analytical Chemistry, vol. 105, pp. 218-224, 2018.

[8] Y. Wu, L. Jiao, X. Luo et al., "Oxidase-like Fe-N-C single-atom nanozymes for the detection of acetylcholinesterase activity," Small, vol. 15, no. 43, article 1903108, 2019.

[9] L. Jiao, W. Xu, H. Yan et al., "Fe-N-C single-atom nanozymes for the intracellular hydrogen peroxide detection," Analytical Chemistry, vol. 91, no. 18, pp. 11994-11999, 2019.

[10] K. Fan, J. Xi, L. Fan et al., "In vivo guiding nitrogen-doped carbon nanozyme for tumor catalytic therapy," Nature Communications, vol. 9, no. 1, p. 1440, 2018.

[11] H. Sun, Y. Zhou, J. Ren, and X. Qu, "Carbon nanozymes: enzymatic properties, catalytic mechanism, and applications," Angewandte Chemie International Edition, vol. 57, no. 30, pp. 9224-9237, 2018.

[12] Y. Hu, X. J. Gao, Y. Zhu et al., "Nitrogen-doped carbon nanomaterials as highly active and specific peroxidase mimics," Chemistry of Materials, vol. 30, no. 18, pp. 64316439, 2018.

[13] P. Zhang, D. Sun, A. Cho et al., "Modified carbon nitride nanozyme as bifunctional glucose oxidase-peroxidase for metal-free bioinspired cascade photocatalysis," Nature Communications, vol. 10, no. 1, p. 940, 2019. 
[14] L. Huang, J. Chen, L. Gan, J. Wang, and S. Dong, "Single-atom nanozymes," Science Advances, vol. 5, no. 5, article eaav5490, 2019.

[15] H. Sun, A. Zhao, N. Gao, K. Li, J. Ren, and X. Qu, “Deciphering a nanocarbon-based artificial peroxidase: chemical identification of the catalytically active and substrate-binding sites on graphene quantum dots," Angewandte Chemie International Edition, vol. 54, no. 24, pp. 7176-7180, 2015.

[16] X. Mu, H. He, J. Wang et al., "Carbogenic nanozyme with ultrahigh reactive nitrogen species selectivity for traumatic brain injury," Nano Letters, vol. 19, no. 7, pp. 4527-4534, 2019.

[17] M. Inagaki, M. Toyoda, Y. Soneda, and T. Morishita, "Nitrogen-doped carbon materials," Carbon, vol. 132, pp. 104-140, 2018.

[18] X. Li, B. Y. Guan, S. Gao, and X. W. Lou, “A general dualtemplating approach to biomass-derived hierarchically porous heteroatom-doped carbon materials for enhanced electrocatalytic oxygen reduction," Energy \& Environmental Science, vol. 12, no. 2, pp. 648-655, 2019.

[19] T. Zhang and T. Asefa, "Heteroatom-doped carbon materials for hydrazine oxidation," Advanced Materials, vol. 31, no. 13, article 1804394, 2019.

[20] C. Hu and L. Dai, "Doping of carbon materials for metal-free electrocatalysis," Advanced Materials, vol. 31, no. 7, article 1804672, 2019.

[21] Y. Jiao, Y. Zheng, K. Davey, and S.-Z. Qiao, "Activity origin and catalyst design principles for electrocatalytic hydrogen evolution on heteroatom-doped graphene," Nature Energy, vol. 1, no. 10, article 16130, 2016.

[22] F. Li, T. Li, C. Sun, J. Xia, Y. Jiao, and H. Xu, "Selenium-doped carbon quantum dots for free-radical scavenging," Angewandte Chemie International Edition, vol. 56, no. 33, pp. 9910-9914, 2017.

[23] K. Gao, B. Wang, L. Tao et al., "Efficient metal-free electrocatalysts from $\mathrm{N}$-doped carbon nanomaterials: mono-doping and co-doping," Advanced Materials, vol. 31, no. 13, article 1805121, 2019.

[24] H. Fu, K. Huang, G. Yang et al., "Synergistic effect of nitrogen dopants on carbon nanotubes on the catalytic selective epoxidation of styrene," ACS Catalysis, vol. 10, no. 1, pp. 129-137, 2020.

[25] D. Guo, R. Shibuya, C. Akiba, S. Saji, T. Kondo, and J. Nakamura, "Active sites of nitrogen-doped carbon materials for oxygen reduction reaction clarified using model catalysts," Science, vol. 351, no. 6271, pp. 361-365, 2016.

[26] Y. Chen, L. Jiao, H. Yan et al., "Hierarchically porous S/N codoped carbon nanozymes with enhanced peroxidase-like activity for total antioxidant capacity biosensing," Analytical Chemistry, vol. 92, no. 19, pp. 13518-13524, 2020.

[27] Q. Li, S. Zhang, L. Dai, and L.-s. Li, "Nitrogen-doped colloidal graphene quantum dots and their size-dependent electrocatalytic activity for the oxygen reduction reaction," Journal of the American Chemical Society, vol. 134, no. 46, pp. 1893218935, 2012.

[28] H. B. Yang, J. Miao, S.-F. Hung et al., "Identification of catalytic sites for oxygen reduction and oxygen evolution in $\mathrm{N}$ doped graphene materials: development of highly efficient metal-free bifunctional electrocatalyst," Science Advances, vol. 2, no. 4, article e1501122, 2016.

[29] N. Wang, B. Lu, L. Li et al., "Graphitic nitrogen is responsible for oxygen electroreduction on nitrogen-doped carbons in alkaline electrolytes: insights from activity attenuation studies and theoretical calculations," ACS Catalysis, vol. 8, no. 8, pp. 6827-6836, 2018.

[30] W. Ding, Z. Wei, S. Chen et al., "Space-confinement-induced synthesis of pyridinic- and pyrrolic-nitrogen-doped graphene for the catalysis of oxygen reduction," Angewandte Chemie International Edition, vol. 52, no. 45, pp. 11755-11759, 2013.

[31] H. Wang, T. Maiyalagan, and X. Wang, "Review on recent progress in nitrogen-doped graphene: synthesis, characterization, and its potential applications," ACS Catalysis, vol. 2, no. 5, pp. 781-794, 2012.

[32] S. Valtueña, N. Pellegrini, L. Franzini et al., "Food selection based on total antioxidant capacity can modify antioxidant intake, systemic inflammation, and liver function without altering markers of oxidative stress," The American Journal of Clinical Nutrition, vol. 87, no. 5, pp. 1290-1297, 2008.

[33] H.-W. Liang, X. Zhuang, S. Brüller, X. Feng, and K. Müllen, "Hierarchically porous carbons with optimized nitrogen doping as highly active electrocatalysts for oxygen reduction," Nature Communications, vol. 5, no. 1, article 4973, 2014.

[34] X. Luo, X. Wei, H. Wang et al., "Secondary-atom-doping enables robust $\mathrm{Fe}-\mathrm{N}-\mathrm{C}$ single-atom catalysts with enhanced oxygen reduction reaction," Nano-Micro Letters, vol. 12, no. 1 , article $163,2020$.

[35] Q. Niu, K. Gao, Q. Tang et al., "Large-size graphene-like porous carbon nanosheets with controllable $\mathrm{N}$-doped surface derived from sugarcane bagasse pith/chitosan for high performance supercapacitors," Carbon, vol. 123, pp. 290298, 2017.

[36] H. Jiang, J. Gu, X. Zheng et al., "Defect-rich and ultrathin N doped carbon nanosheets as advanced trifunctional metalfree electrocatalysts for the ORR, OER and HER," Energy \& Environmental Science, vol. 12, no. 1, pp. 322-333, 2019.

[37] B. Jiang, D. Duan, L. Gao et al., "Standardized assays for determining the catalytic activity and kinetics of peroxidase-like nanozymes," Nature Protocols, vol. 13, no. 7, pp. 1506-1520, 2018.

[38] M. S. Kim, S. Cho, S. H. Joo et al., "N- and B-codoped graphene: a strong candidate to replace natural peroxidase in sensitive and selective bioassays," ACS Nano, vol. 13, no. 4, pp. 4312-4321, 2019.

[39] X. Xia, J. Zhang, N. Lu et al., "Pd-Ir core-shell nanocubes: a type of highly efficient and versatile peroxidase mimic," ACS Nano, vol. 9, no. 10, pp. 9994-10004, 2015.

[40] S. Li, L. Shang, B. Xu et al., "A nanozyme with photo-enhanced dual enzyme-like activities for deep pancreatic cancer therapy," Angewandte Chemie International Edition, vol. 58, no. 36, pp. 12624-12631, 2019.

[41] M. S. Kim, J. Lee, H. S. Kim et al., "Heme cofactor-resembling $\mathrm{Fe}-\mathrm{N}$ single site embedded graphene as nanozymes to selectively detect $\mathrm{H}_{2} \mathrm{O}_{2}$ with high sensitivity," Advanced Functional Materials, vol. 30, no. 1, article 1905410, 2020.

[42] L. Gao, J. Zhuang, L. Nie et al., "Intrinsic peroxidase-like activity of ferromagnetic nanoparticles," Nature Nanotechnology, vol. 2, no. 9, pp. 577-583, 2007.

[43] G. I. Berglund, G. H. Carlsson, A. T. Smith, H. Szöke, A. Henriksen, and J. Hajdu, "The catalytic pathway of horseradish peroxidase at high resolution," Nature, vol. 417, no. 6887, pp. 463-468, 2002.

[44] H. Wang, G. Pu, S. Devaramani et al., "Bimodal electrochemiluminescence of G-CNQDs in the presence of double 
coreactants for ascorbic acid detection," Analytical Chemistry, vol. 90, no. 7, pp. 4871-4877, 2018.

[45] Y. Ma, M. Zhao, B. Cai, W. Wang, Z. Ye, and J. Huang, "3D graphene foams decorated by $\mathrm{CuO}$ nanoflowers for ultrasensitive ascorbic acid detection," Biosensors and Bioelectronics, vol. 59, pp. 384-388, 2014.

[46] Q. Chen, S. Li, Y. Liu et al., "Size-controllable Fe-N/C singleatom nanozyme with exceptional oxidase-like activity for sensitive detection of alkaline phosphatase," Sensors and Actuators B: Chemical, vol. 305, article 127511, 2020.

[47] G. Kresse and J. Hafner, "Ab initiomolecular dynamics for open-shell transition metals," Physical Review B, vol. 48, no. 17, pp. 13115-13118, 1993.

[48] G. Kresse and J. Furthmüller, "Efficient iterative schemes for ab initio total-energy calculations using a plane-wave basis set," Physical Review B, vol. 54, no. 16, pp. 11169-11186, 1996.

[49] P. E. Blöchl, "Projector augmented-wave method," Physical Review B, vol. 50, no. 24, pp. 17953-17979, 1994.

[50] J. P. Perdew, K. Burke, and M. Ernzerhof, "Generalized gradient approximation made simple," Physical Review Letters, vol. 77, no. 18, pp. 3865-3868, 1996.

[51] R. Bader, "Atoms in molecules: a quantum theory," Journal of Molecular Structure: THEOCHEM, vol. 360, 1996. 